\title{
ASSAY OF ANTIOXIDANT POTENTIAL OF TWO ASPERGILLUS ISOLATES BY DIFFERENT METHODS UNDER VARIOUS PHYSIO-CHEMICAL CONDITIONS
}

\author{
Daljit Singh Arora*, Priyanka Chandra
}

Microbial Technology Laboratory, Department of Microbiology, Guru Nanak Dev University, Amritsar-143005, India.

\begin{abstract}
The objective of this work was to screen fungi isolated from soil of different areas of Punjab, India for antioxidant activity by dot blot assay and around $45 \%$ of fungal isolates demonstrated antioxidant potential. Two selected strains of Aspergillus spp (Aspergillus PR78 and Aspergillus PR66) showing quantitatively best antioxidant activity by DPPH assay were further tested for their reducing power, ferrous ion and nitric oxide ion scavenging activity, FRAP assay and total phenolic content. Different physio-chemical parameters were optimized for enhancement of the activity. This revealed stationary culture grown for 10 days at $25{ }^{\circ} \mathrm{C}$ at $\mathrm{pH} 7$ to be the best for antioxidant activity. Sucrose in the medium as carbon source resulted in highest antioxidant activity. Sodium nitrate, yeast extract, and peptone were good sources of nitrogen but sodium nitrate was the best among these. The extraction of the broth culture filtrates with different solvents revealed ethyl acetate extract to possess the best antioxidant activity. The activity as expressed by ethyl acetate extract of Aspergillus PR78 was equally effective as that of commonly used antioxidant standard, ascorbic acid.
\end{abstract}

Key words: Antioxidant potential; Aspergillus; Dot blot assay; DPPH assay; FRAP assay; Reducing power; Soil fungi.

\section{INTRODUCTION}

Biotechnology consists in the use of cellular systems for the development of processes and products holding economical and social relevance to humankind. The fungi are of great biotechnological interest in the fermentative processes that culminate in the production of secondary metabolites (19). Filamentous fungi produce a diverse array of secondary metabolites, the small molecules that are not necessary for normal growth or development (11). Secondary metabolites have a tremendous impact on society and are exploited for their antibiotics and pharmaceutical activities such as anticancer, antitumor, immuno-stimulatory, and antioxidants (6). The polyphenolic compounds are the secondary metabolites commonly found in plants, mushrooms and fungi and have been reported to possess multiple biological effects such as anti-inflammatory, antiarteriosclerotic, antitumor, antimutagenic, anticarcinogenic, antibacterial and cardio protective actions including antioxidant activity (15).

Free radicals are implicated in the pathogenesis of various human diseases such as arteriosclerosis, cancer, diabetes mellitus, liver injury, inflammation, skin damages, coronary 
heart diseases, and arthritis (17). Antioxidants serve as the defensive factor against free radicals in the body. Synthetic antioxidants such as butylated hydroxyanisole (BHA), butylated hydroxytoluene (BHT) and tert-butylhydroquinone (TBHQ) are usually used as food additives by the food industry to prevent lipid peroxidation. However, their application has been limited because of possible toxic and carcinogenic components formed during their degradation. In view of these health concerns finding safer, more effective and economic natural antioxidants is highly desirable (13). A number of plants and mushrooms (fruiting body) are commonly known to produce antioxidants but there are few reports on lower fungi (21). These include Penicillium roquefortii, Aspergillus candidus, Mortierella, Emericella falconensis, Acremonium, Colletotrichum gloeosporioides (21), Mycelia sterilia (17), Antrodia camphorata (23), Chaetomium sp., Cladosporium sp., Torula sp., Phoma sp. etc (9). A lot more fungi still needs to be explored as the production, downstream processing of actual bioactive phytochemicals from plants is quite tougher as compared to microbes. Keeping above in mind the present study was planned to screen and expand the spectrum of fungi having antioxidant potential and to optimize the culture conditions to enhance the activity.

\section{MATERIALS AND METHODS}

\section{Experimental}

Fungi were isolated from soils of different regions of Punjab. The fungal isolates so obtained were maintained by regular subculturing on yeast extract glucose agar (YGA) slants and stored at $4^{\circ} \mathrm{C}$. The cultures were also preserved in $10 \%$ glycerol at $-70^{\circ} \mathrm{C}$. The isolated fungi were grown on YGA plates for 6-7 days from which two discs $(8 \mathrm{~mm})$ of fungal mycelia were used to inoculate $25 \mathrm{ml}$ Czapek dox's broth (sucrose $3 \%$, sodium nitrate $0.2 \%, \mathrm{~K}_{2} \mathrm{HPO}_{4} 0.1 \%$, magnesium sulphate $0.05 \%$, potassium chloride $0.05 \%$, ferrous sulphate $0.001 \%$ ). After incubation of 10 days at static conditions (25 ${ }^{\circ} \mathrm{C}$ ), the culture broth of each fungus was filtered through
Whatman filter paper no. 1 and the filtrate so obtained was used for different assay procedures.

Screening of the soil fungi for antioxidant activity by dotblot 1,1-diphenyl -2-picryl hydrazyl (DPPH) assay

Initially, all the 113 fungi isolated from soil were screened for their antioxidant activity according to dot blot DPPH rapid staining method (8). Drops taken from ten days grown fungal cultures were spotted onto TLC plates (Silica gel $60 \mathrm{~F}_{254}$ ) with the help of micro capillary and allowed to dry for 3 min. The staining of silica gel TLC plates was carried out by placing them upside down for $10 \mathrm{sec}$ in $0.4 \mathrm{mM}$ DPPH solution. The excess solution was removed with a blotting paper and the plates were air-dried. Stained silica layers revealed a purple background with white spots indicating radical scavenging capacity of the organisms. The intensity of the white color depends upon the amount and nature of radical scavengers present in the sample. Ascorbic acid was taken as the standard and distilled water was taken as negative control.

\section{Quantitative assay for DPPH free radicals scavenging activity}

The scavenging activity for DPPH free radicals was measured according to Zhao et al. (29). An aliquot of $1 \mathrm{ml}$ of $0.1 \mathrm{mM}$ DPPH solution in ethanol and $0.5 \mathrm{ml}$ of extract were mixed. The mixture was shaken vigorously and allowed to reach a steady state at room temperature for $30 \mathrm{~min}$. Decolourization of DPPH was determined by measuring the decrease in absorbance at $517 \mathrm{~nm}$, and the DPPH radical scavenging effect was calculated according to the following equation:

$$
\% \text { scavenging rate }=[1-(\mathrm{A} 1-\mathrm{A} 2) / \mathrm{A} 0] \times 100
$$

Where $\mathrm{A} 0$ represents the absorbance of the control and A1 represents the absorbance of extract, A2 represents the absorbance without DPPH.

\section{Determination of antioxidant activity by reducing power measurement}

The reducing power of the extracts was determined 
according to Chang et al. (5). An aliquot of $0.5 \mathrm{ml}$ extract was added to $0.1 \mathrm{ml}$ of $1 \%$ potassium ferricyanide. After incubating the mixture at $50{ }^{\circ} \mathrm{C}$ for $30 \mathrm{~min}$, during which ferricyanide was reduced to ferrocyanide, it was supplemented with $0.1 \mathrm{ml}$ of $1 \%$ trichloroacetic acid and $0.1 \% \mathrm{FeCl}_{3}$, and left for $20 \mathrm{~min}$. Absorbance was read at $700 \mathrm{~nm}$ to determine the amount of ferric ferrocyanide (Prussian blue) formed. Higher absorbance of the reaction mixture indicates higher reducing power of the sample.

\section{Determination of antioxidant activity by ferric reducing antioxidant power (FRAP) assay}

FRAP assay was carried out according to Othman et al. (20) by monitoring the reduction of $\mathrm{Fe}^{3+}$ - tripyridyl triazine (TPTZ) to blue colored $\mathrm{Fe}^{2+}$-TPTZ. The FRAP reagent was prepared by mixing $300 \mathrm{mM}$ acetate buffer (pH 3.6), $10 \mathrm{mM}$ TPTZ and $20 \mathrm{mM}$ ferric chloride in a ratio of 10:1:1. The reaction mixture containing $2 \mathrm{ml}$ of FRAP reagent, $0.5 \mathrm{ml}$ of extract and $1 \mathrm{ml}$ of distilled water was incubated for $10 \mathrm{~min}$ and the absorbance measured at $593 \mathrm{~nm}$. Antioxidant potential of the sample was compared with the activity of $0.5 \mathrm{ml}$ stock solution of $1 \mathrm{mg} / \mathrm{ml} \mathrm{FeSO}_{4}$.

\section{Determination of ferrous ion scavenging (metal chelating) activity}

The chelating activity of the extracts for ferrous ions was measured according to Zhao et al. (29). The reaction mixture containing $0.5 \mathrm{ml}$ of extract, $1.6 \mathrm{ml}$ of deionized water, $0.05 \mathrm{ml}$ of $\mathrm{FeCl}_{2}(2 \mathrm{mM})$ and $0.1 \mathrm{ml}$ of ferrozine $(5 \mathrm{mM})$ was incubated at $40{ }^{\circ} \mathrm{C}$ for $10 \mathrm{~min}$ and the absorbance measured at $562 \mathrm{~nm}$. The chelating activity was calculated as:

$$
\text { Chelating rate }=[1-(\mathrm{A} 1-\mathrm{A} 2) / \mathrm{A} 0] \times 10
$$

Where $\mathrm{A} 0$ represents the absorbance of the control and A1 represents the absorbance of extract, A2 represents the absorbance without $\mathrm{FeCl}_{2}$.

\section{Determination of nitric oxide (NO) scavenging activity}

Nitric oxide production from sodium nitroprusside was measured according to Kang et al. (10). An equal amount (6 $\mathrm{ml})$ of sodium nitroprusside $(5 \mathrm{mM})$ solution was mixed with 6 $\mathrm{ml}$ of extract and incubated at $25{ }^{\circ} \mathrm{C}$ for two and half hours. After every half an hour, $0.5 \mathrm{ml}$ of the reaction mixture was mixed with an equal amount of Griess reagent $(1 \%$ sulphanilamide, $2 \%$ phosphoric acid, and $0.1 \%$ napthylethylene diamine dihydrochloride) and absorbance was taken at $546 \mathrm{~nm}$ and compared with absorbance of $1 \mathrm{mg} / \mathrm{ml}$ of standard solution (sodium nitrite) treated in the same way with Griess reagent.

\section{Determination of total phenolic content (TPC)}

The total polyphenolic content was determined colorimetrically using the Folin-Ciocalteau (FC) method according to Singleton et al. (22) with some modifications. Test sample $(0.5 \mathrm{ml})$ was mixed with $0.2 \mathrm{ml}$ of $\mathrm{FC}$ reagent and allowed to stand for $10 \mathrm{~min}$ to which $0.6 \mathrm{ml}$ of $20 \%$ sodium carbonate was added and mixed completely. The reaction mixture was incubated at $40^{\circ} \mathrm{C}$ for $30 \mathrm{~min}$. Absorbance of the reaction mixture was measured at $765 \mathrm{~nm}$. Gallic acid was taken as standard.

\section{Optimization of physio-chemical and nutritional parameters}

To optimize the physio-chemical and nutritional parameters to express the best antioxidant potential, both the organisms were grown at different temperature and $\mathrm{pH}$ values, static as well as shaking conditions. Time profile for antioxidant potential was monitored by assaying the antioxidant activity by different procedures on every $5^{\text {th }}$ day upto 30 days of growth under static conditions.

\section{Effect of carbon and nitrogen sources}

To find the best carbon source, sucrose in the Czapek dox's medium was replaced with different sugars (glucose, maltose, lactose, and starch) while to work out the best nitrogen source, sodium nitrate was replaced with different nitrogen sources (yeast extract, peptone, malt extract, and casein) at same concentration i.e. $3 \%$ and $0.2 \%$, respectively.

\section{Thermostability of antioxidant bioactivity}

To check the temperature sensitivity of the culture broth 
for antioxidant activity, it was subjected to $40^{\circ} \mathrm{C}, 60^{\circ} \mathrm{C}, 80^{\circ} \mathrm{C}$, $100^{\circ} \mathrm{C}$ for one $\mathrm{hr}$ and the heat treated broth was then assayed for the residual antioxidant activity.

\section{Effect of different organic solvents}

To work out the best organic solvent for extraction of bioactive component, the culture broth was treated with different solvents viz petroleum ether, chloroform, ethyl acetate and butanol. Solvents extracted components were then evaporated to dryness in vaccuo and the resulting solids were reconstituted in methanol to get five times concentrated stock. Then this stock was checked for their antioxidant potential by various assays.

\section{Statistical analysis}

All the tests were performed in triplicate. The results are expressed as mean \pm SD values. Pearson's correlation coefficient was also analyzed. $p$ value less than 0.05 was considered statistically significant.

\section{RESULTS}

\section{Rapid screening of antioxidant activity by dot blot assay}

Antioxidant capacity of fungal extracts was detected semiquantitatively by a rapid DPPH staining method. This method is typically based on the inhibition of the accumulation of oxidized products, since the generation of free radicals is inhibited by the addition of antioxidants and scavenging of the free radicals shifts the end point. The appearance of a white color spot against purple background is of much screening value for the evaluation of antioxidant potential of the organism. Out of 113 fungal isolates, 51 showed white colored spot against purple background. The intensity of white color was however variable for different organisms. The two best strains selected on the basis of quantitative DPPH assay and identified as Aspergillus were selected for further studies and quantification.

\section{Comparison of antioxidant potential by different quantitative methods}

The different assay procedures demonstrated both
Aspergillus spp. to possess potent antioxidant potential while Aspergillus PR78 was better in comparison to Aspergillus PR66. The DPPH radicals are widely used to investigate the scavenging activities and both the organisms showed a good scavenging effect on DPPH radicals but Aspergillus PR78 $(82.77 \%)$ was significantly stronger scavenger than Aspergillus PR66 (68.26\%). In order to examine the reducing power of two organisms the reduction of $\mathrm{Fe}^{3+}$ to $\mathrm{Fe}^{2+}$ was carried out by culture extracts of 2 organisms, whereas Aspergillus PR78 (1.36) exhibited strong reducing power than Aspergillus PR66 (1.09). Similarly Aspergillus PR78 and Aspergillus PR66 demonstrated effective ferric ion reduction based on FRAP assay. Aspergillus PR78 was more effective than Aspergillus PR66 and gave reduction rate of $64.2 \%$ and $52.22 \%$, respectively. In addition, the chelating activities for ferrous ion were assayed for both Aspergillus spp. Aspergillus PR78 and Aspergillus PR66 chelated $68.23 \%$ and $53.03 \%$ of ferrous ion, respectively. Nitric oxide ion scavenging is also essential with other scavenging activity as NO is a very reactive ion and results showed that both the strains exhibited inhibition of formation of NO. The percentage rate of scavenging nitric oxide ion for Aspergillus PR78 and Aspergillus PR66 are $64.4 \%$ and $51.32 \%$, respectively.

\section{Total phenolic content}

The TPC of Aspergillus extracts have been expressed as gallic acid equivalent (GAE) i.e. $\mathrm{mg}$ gallic acid $/ 100 \mathrm{ml}$ culture. TPC are known to be responsible for antioxidant activity and both the Aspergillus spp. possessed high TPC, which is positively correlated with their antioxidant potential. The TPC of Aspergillus PR78 (16.74 mg/ml) was higher than Aspergillus PR66 (13.05 mg/ml).

\section{Antioxidant activity under different physio-chemical} conditions

Effect of shaking condition: The experiments carried out to see the effect of shaking at different RPM demonstrated static culture to give better antioxidant yield in comparison to shake flask cultures, which resulted in steady decline in the 
activity with increase in RPM. Thus, further optimization was carried out under static condition (Table 1).

Effect of growth period: The antioxidant potential measured by different assay procedures was best expressed on $10^{\text {th }}$ day, which subsequently declined up to 30 days (Fig 1).
Effect of temperature and pH: The antioxidant potential with different assay procedures demonstrated the best activity at $25^{\circ} \mathrm{C}$ (Fig 2) and between $\mathrm{pH} 5-7$. No activity could be detected at extreme $\mathrm{pH}$ values $(2,3,10,11$, and 12$)$ by any of the methods (Fig 3).

Table 1. Effect of shaking on antioxidant potential of two Aspergillus spp.

\begin{tabular}{|c|c|c|c|c|c|}
\hline \multirow{2}{*}{$\%$ activity } & & \multicolumn{2}{|c|}{ Static condition } & \multicolumn{2}{|c|}{ Shaking condition (100 RPM) } \\
\hline & & PR 78 & PR 66 & PR78 & PR66 \\
\hline DPPH assay & & $82.77 \pm 0.05$ & $68.26 \pm 0.1$ & $66.41 \pm 0.2$ & $59.77 \pm 0.09$ \\
\hline Reducing power & & $1.36 \pm 0.20$ & $1.09 \pm 0.08$ & $1.01 \pm 0.09$ & $0.911 \pm 0.09$ \\
\hline $\begin{array}{l}\mathrm{Fe}^{2+} \text { scavenging } \\
\text { Activity }\end{array}$ & & $68.23 \pm 0.13$ & $53.03 \pm 0.05$ & $60.3 \pm 0.02$ & $40.2 \pm 0.8$ \\
\hline FRAP assay & & $64.2 \pm 0.25$ & $52.22 \pm 0.44$ & $58.4 \pm 0.32$ & $38.4 \pm 0.3$ \\
\hline \multirow{5}{*}{$\begin{array}{l}\text { NO scavenging } \\
\text { activity }\end{array}$} & $30 \mathrm{~min}$ & $33.4 \pm 0.54$ & $15.01 \pm 0.21$ & $8.6 \pm 0.2$ & $24.4 \pm 0.22$ \\
\hline & $60 \mathrm{~min}$ & $42.3 \pm 0.07$ & $22.8 \pm 0.31$ & $15.4 \pm 0.08$ & $30.2 \pm 0.15$ \\
\hline & $90 \mathrm{~min}$ & $50.4 \pm 0.19$ & $32.06 \pm 0.36$ & $20.8 \pm 0.09$ & $39.4 \pm 0.32$ \\
\hline & $120 \mathrm{~min}$ & $58.3 \pm 0.26$ & $42.98 \pm 0.34$ & $24.3 \pm 0.06$ & $47.3 \pm 0.23$ \\
\hline & $180 \mathrm{~min}$ & $64.4 \pm 0.17$ & $51.32 \pm 0.23$ & $32.4 \pm 0.4$ & $52.4 \pm 0.13$ \\
\hline TPC (mg/ml) & & $16.74 \pm 0.02$ & $13.05 \pm 0.08$ & $12.3 \pm 0.02$ & $8.6 \pm 0.3$ \\
\hline
\end{tabular}

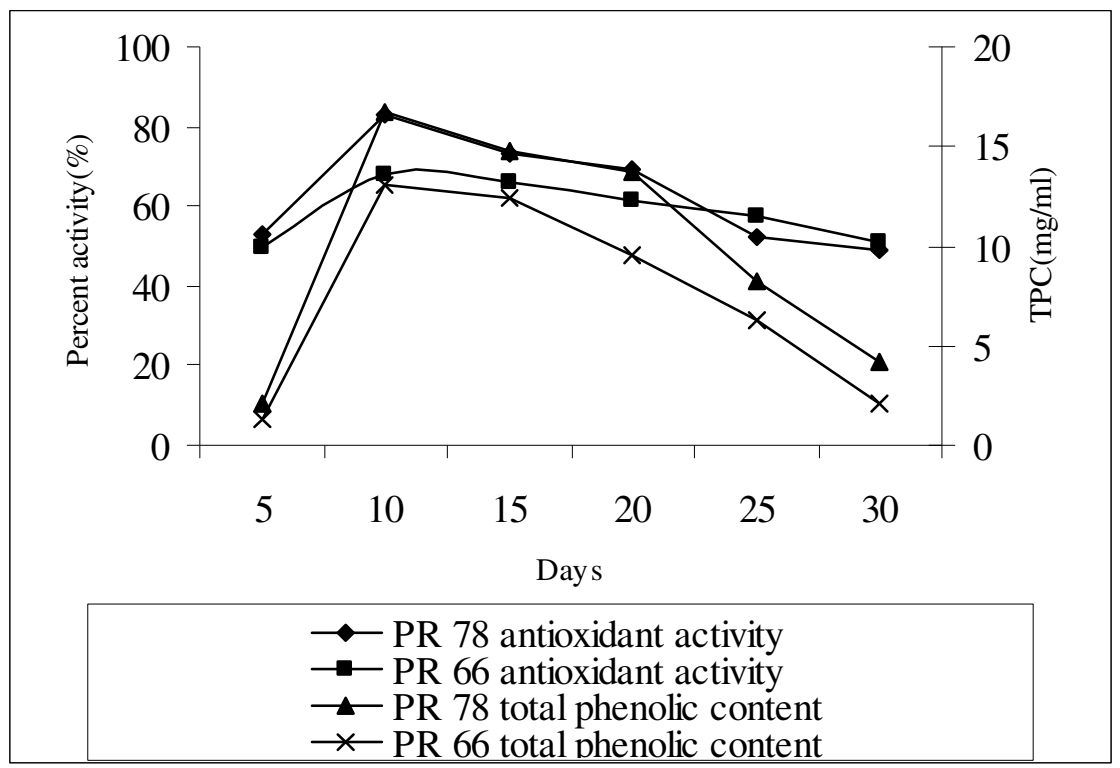

Figure 1. Antioxidant activity and total phenolic content of two Aspergillus spp grown as static culture during different incubation periods. 


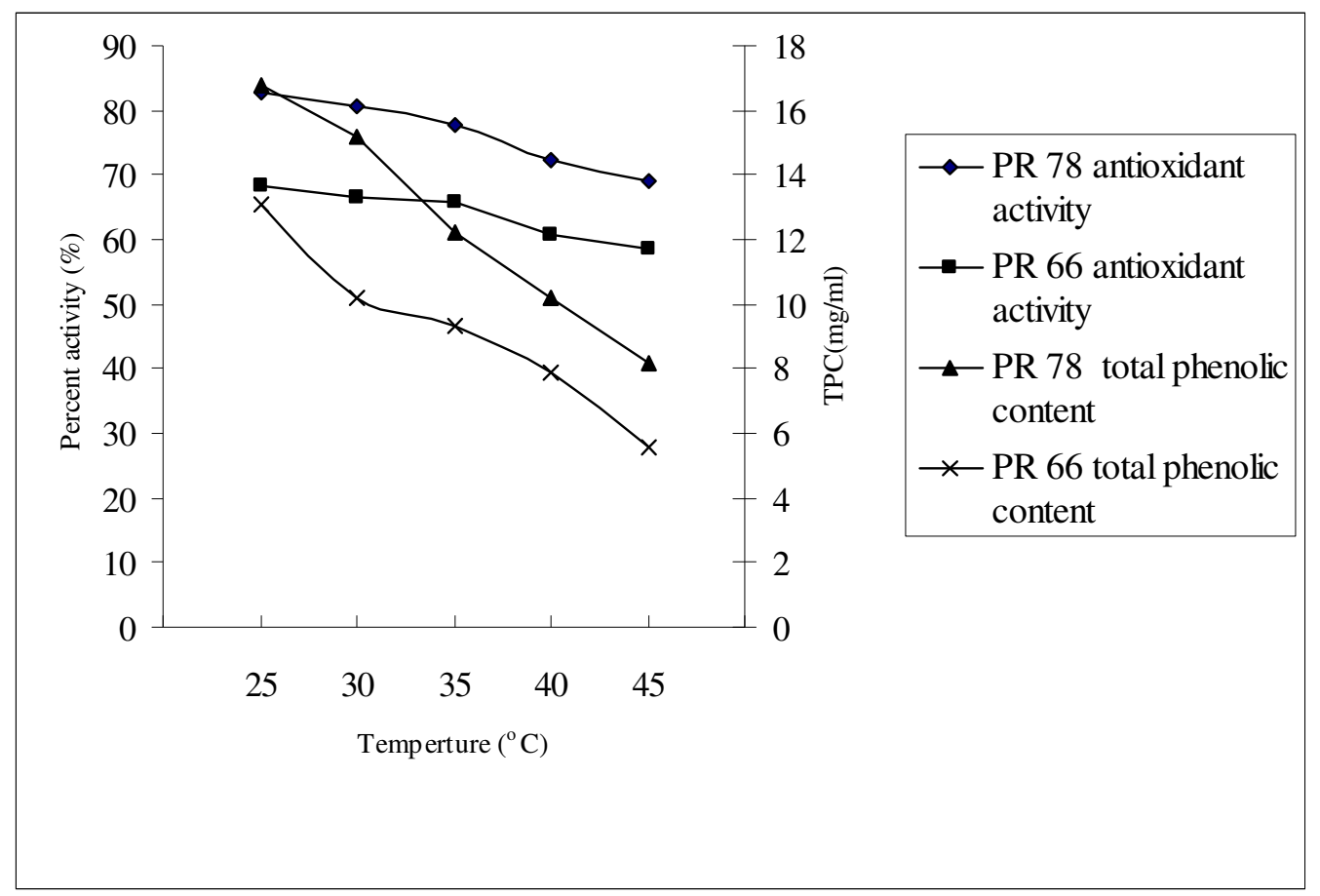

Figure 2. Effect of temperature on antioxidant activity and total phenolic content of two Aspergillus spp grown as static culture.

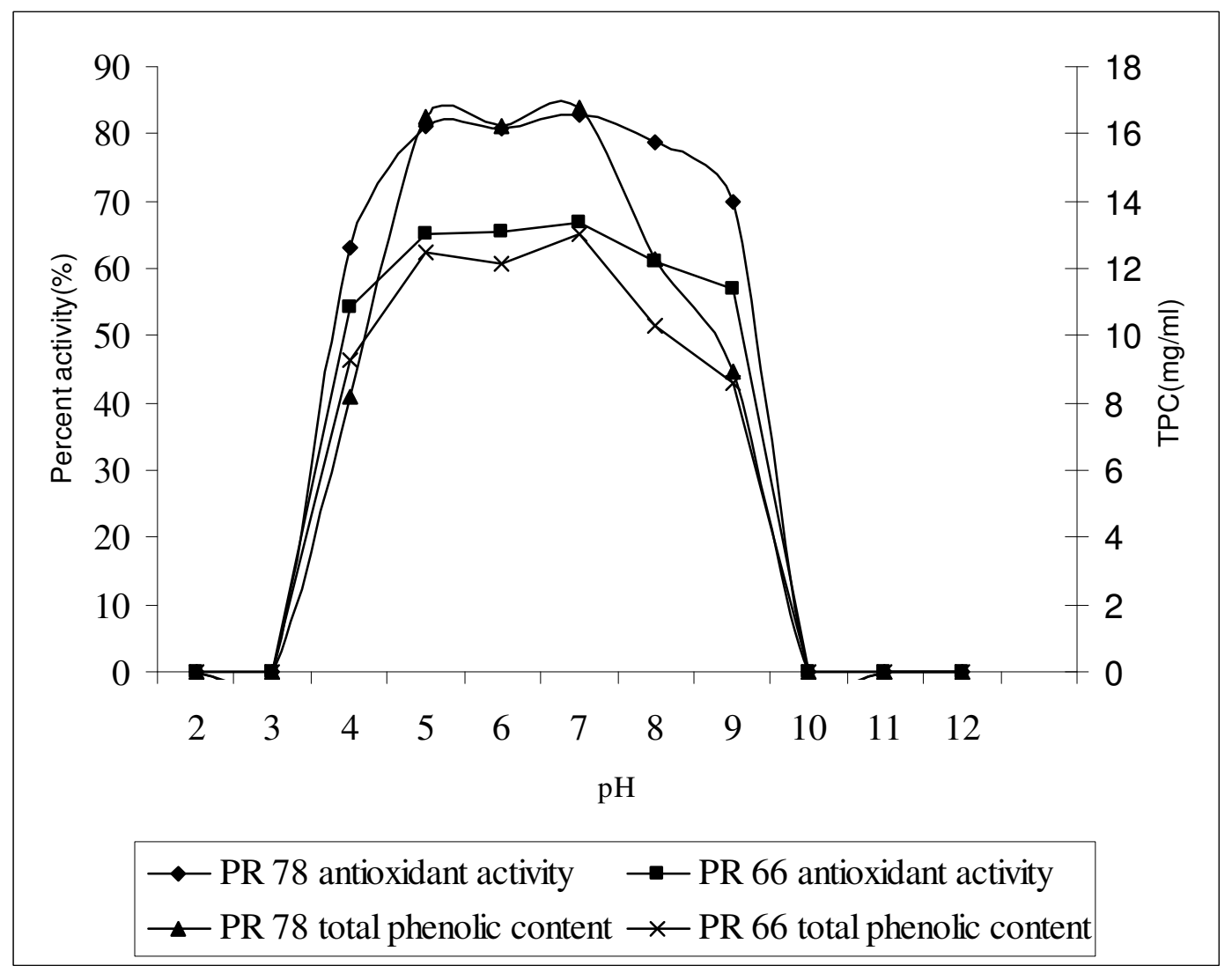

Figure 3. Effect of $\mathrm{pH}$ on the antioxidant activity and total phenolic content of two Aspergillus spp grown as static culture. 
Effect of carbon and nitrogen sources: The antioxidant activity was best expressed in sucrose as carbon source (Table 2 ) and the order of activity under various other carbon sources was as follows sucrose $>$ dextrose $>$ maltose $>$ lactose $>$ starch. Similarly sodium nitrate was best among inorganic as well as organic nitrogen sources. Though peptone and yeast extract were also good source of nitrogen. The order of various nitrogen sources showing activity was as follows sodium nitrate $>$ yeast extract $>$ peptone $>$ casein $>$ malt extract (Table 3 ).

\section{Thermostability of antioxidant bioactivity:}

Thermostability of the active components in the culture filtrate responsible for antioxidant activity was checked and found to be relatively thermostable. The antioxidant activity decreased with the increase of heat exposure. At $40^{\circ} \mathrm{C}$ the activity decreased by only $3-27 \%$ in both the organisms, while at 100 ${ }^{\circ} \mathrm{C}$ it suffered a maximum loss of $50 \%$ in its activity (Fig 4).

Effect of different organic solvents: The extraction with different solvents revealed ethyl acetate to be the best solvent to elute the components responsible for antioxidant potential that was followed by chloroform and butanol extract. Petroleum ether extracts did not show any activity. The activity exhibited by the broth extracted in different solvents indicates that there may be many components with different polarities present in the broth (Table 4).

\section{DISCUSSION}

Vast diversity of microbes remain untapped for structurally diverse metabolites possessing highly valuable bioactivities including antioxidant activity (18). In the present study, 45 percent of soil fungal isolates demonstrated their antioxidant potential to variable levels. Qualitative test proved successful for bulk screening of samples though quantitative analysis revealed the differential ability of such fungi. Based on these observations the two best strains of Aspergillus spp were selected for further studies. Aspergillus PR78 was found to have overall better antioxidant activity than Aspergillus
PR66. The yield of bioactive metabolite can sometime be substantially increased by the optimization of physical and chemical factors used for the growth of microbes; a good understanding of the role of these factors in the biosynthesis of metabolites may lead to better exploitation (28).

The comparative analysis of the data obtained for two Aspergillus spp for their antioxidant potential assayed by different methods demonstrated static culture conditions to be more suitable as compared to shake flask. It might be attributed to low amount of phenolic compounds produced under shaking conditions, which have been held responsible for antioxidant activity of fungi. This supports the earlier contention of various researchers who have used static conditions (7) or low RPM (between 100 to $150 \mathrm{RPM})(9,12,25)$. The optimum period of incubation for antioxidant potential of the isolates was found to be 10 day and subsequent decline in bioactivity could be due to the exhaustion of nutrients available for the fungi. Alternatively, may be due to the degradation of secondary metabolites (phenolic compounds) already produced by fungi as also revealed from the decline in the amount of phenolic content.

The comparison of antioxidant potential in the culture extract obtained from two fungi grown at different temperatures $25^{\circ} \mathrm{C}$ revealed to be the optimum for both the organisms, which correlate positively with their phenolic content. No activity was detected at $\mathrm{pH}$ extremes, which was optimally best between the $\mathrm{pH}$ 5-7. The present results corroborate the previous studies in which there was no bioactivity at $\mathrm{pH}$ extremes (14). This may be due to delayed metabolite production caused by delayed mycelial growth or due to a reduced production of bioactive metabolites under such $\mathrm{pH}$ conditions. This shows that $\mathrm{pH}$ of the growth medium can also significantly effect the production of secondary metabolites. The $\mathrm{pH}$ is related to permeability characteristics of the cell wall and membrane, thus affect either ion uptake or loss to the nutrient medium. 
Table 2. Effect of various carbon sources on antioxidant potential of two Aspergillus spp.

\begin{tabular}{|c|c|c|c|c|c|c|c|c|c|c|c|}
\hline \multirow{2}{*}{\multicolumn{2}{|c|}{$\%$ activity }} & \multicolumn{2}{|l|}{ Dextrose } & \multicolumn{2}{|l|}{ Maltose } & \multicolumn{2}{|l|}{ Lactose } & \multicolumn{2}{|l|}{ Starch } & \multicolumn{2}{|l|}{ Sucrose } \\
\hline & & PR78 & PR66 & PR78 & PR66 & PR78 & PR66 & PR78 & PR66 & PR78 & PR66 \\
\hline DPPH Assay & & $62.48 \pm 0.14$ & $59.89 \pm 0.09$ & $60.39 \pm 0.34$ & $62.84 \pm 0.08$ & $54.58 \pm 0.8$ & $62.84 \pm 0.45$ & $50.30 \pm 0.05$ & $55.6 \pm 0.02$ & $82.77 \pm 0.05$ & $68.26 \pm 0.1$ \\
\hline \multicolumn{2}{|c|}{ Reducing power } & $1.2 \pm 0.32$ & $1.04 \pm 0.20$ & $1.06 \pm 0.13$ & $1.01 \pm 0.23$ & $0.911 \pm 0.29$ & $0.632 \pm 0.43$ & $0.932 \pm 0.34$ & $0.913 \pm 0.32$ & $1.36 \pm 0.20$ & $1.09 \pm 0.08$ \\
\hline \multicolumn{2}{|c|}{$\begin{array}{l}\mathrm{Fe}^{2+} \text { scavenging } \\
\text { activity }\end{array}$} & $59.3 \pm 0.30$ & $50.2 \pm 0.31$ & $57.2 \pm 0.11$ & $49.89 \pm 0.41$ & $49.3 \pm 0.31$ & $34.2 \pm 0.90$ & $50.3 \pm 0.72$ & $39.2 \pm 0.45$ & $68.23 \pm 0.13$ & $53.03 \pm 0.05$ \\
\hline \multicolumn{2}{|l|}{ FRAP assay } & $54.2 \pm 0.18$ & $51.30 \pm 0.12$ & $52.4 \pm 0.17$ & $50.8 \pm 0.06$ & $43.4 \pm 0.09$ & $38.0 \pm 0.35$ & $45.4 \pm 0.60$ & $40.68 \pm 0.92$ & $64.2 \pm 0.25$ & $52.22 \pm 0.44$ \\
\hline \multirow{5}{*}{$\begin{array}{l}\text { NO } \\
\text { scavenging } \\
\text { activity }\end{array}$} & $30 \mathrm{~min}$ & $20.9 \pm 0.02$ & $12.30 \pm 0.09$ & $19.2 \pm 0.03$ & $12.2 \pm 0.99$ & $15.3 \pm 0.21$ & $10.2 \pm 0.33$ & $10.3 \pm 0.03$ & $10.2 \pm 0.23$ & $33.4 \pm 0.54$ & $15.01 \pm 0.21$ \\
\hline & $60 \mathrm{~min}$ & $32.4 \pm 0.01$ & $20.8 \pm 0.15$ & $31.8 \pm 0.13$ & $21.3 \pm 0.11$ & $22.9 \pm 0.07$ & $16.4 \pm 0.61$ & $26.2 \pm 0.22$ & $16.4 \pm 0.31$ & $42.3 \pm 0.07$ & $22.8 \pm 0.31$ \\
\hline & $90 \mathrm{~min}$ & $40.3 \pm 0.03$ & $28.2 \pm 0.32$ & $39.2 \pm 0.23$ & $26.8 \pm 0.37$ & $30.4 \pm 0.41$ & $18.2 \pm 0.31$ & $33.4 \pm 0.42$ & $20.2 \pm 0.65$ & $50.4 \pm 0.19$ & $32.06 \pm 0.36$ \\
\hline & $120 \mathrm{~min}$ & $48.6 \pm 0.01$ & $39.2 \pm 0.22$ & $45.3 \pm 0.45$ & $34.2 \pm 0.30$ & $36.3 \pm 0.08$ & $28.3 \pm 0.42$ & $38.2 \pm 0.30$ & $25.3 \pm 0.36$ & $58.3 \pm 0.26$ & $42.98 \pm 0.34$ \\
\hline & $180 \mathrm{~min}$ & $54.4 \pm 0.07$ & $50.3 \pm 0.50$ & $50.3 \pm 0.56$ & $49.6 \pm 0.26$ & $40.2 \pm 0.25$ & $30.6 \pm 0.21$ & $40.3 \pm 0.39$ & $28.2 \pm 0.22$ & $64.4 \pm 0.17$ & $51.32 \pm 0.23$ \\
\hline \multicolumn{2}{|l|}{$\mathrm{TPC}(\mathrm{mg} / \mathrm{ml})$} & $11.06 \pm 0.21$ & $11.93 \pm 0.40$ & $8.59 \pm 0.30$ & $12.95 \pm 0.23$ & $3.34 \pm 0.45$ & $6.4 \pm 0.6$ & $5.78 \pm 0.087$ & $9.26 \pm 0.02$ & $16.74 \pm 0.02$ & $13.05 \pm 0.08$ \\
\hline
\end{tabular}

DPPH-1.1-diphenyl -2-picryl hydrazyl; FRAP- Ferric reducing antioxidant power; NO-nitric oxide; TPC-total phenolic content 
Table 3. Effect of various nitrogen sources on antioxidant potential of two Aspergillus spp.

\begin{tabular}{|c|c|c|c|c|c|c|c|c|c|c|}
\hline \multirow[b]{2}{*}{ \%activity } & \multicolumn{2}{|c|}{ Yeast extract } & \multicolumn{2}{|c|}{ Peptone } & \multicolumn{2}{|c|}{ Malt extract } & \multicolumn{2}{|c|}{ Casein } & \multicolumn{2}{|c|}{ Sodium nitrate } \\
\hline & PR78 & PR66 & PR78 & PR66 & PR78 & PR66 & PR78 & PR66 & PR78 & PR66 \\
\hline DPPH Assay & $73.39 \pm 0.05$ & $66.17 \pm 0.2$ & $69.73 \pm 0.04$ & $65.55 \pm 0.02$ & $69.0 \pm 0.3$ & $62.0 \pm 0.011$ & $70.1 \pm 0.01$ & $60.38 \pm 0.02$ & $82.77 \pm 0.05$ & $68.26 \pm 0.1$ \\
\hline Reducing power & $1.26 \pm 0.32$ & $1.01 \pm 0.8$ & $1.13 \pm 0.65$ & $1.1 \pm 0.3$ & $0.792 \pm 0.45$ & $0.616 \pm 0.02$ & $1.1 \pm 0.09$ & $0.974 \pm 0.03$ & $1.36 \pm 0.20$ & $1.09 \pm 0.08$ \\
\hline $\begin{array}{c}\mathrm{Fe}^{2+} \text { scavenging } \\
\text { activity }\end{array}$ & $64.5 \pm 0.4$ & $50.2 \pm 0.02$ & $65.3 \pm 0.8$ & $50.8 \pm 0.52$ & $47.7 \pm 0.05$ & $20.2 \pm 0.32$ & $40.2 \pm 0.4$ & $32.3 \pm 0.001$ & $68.23 \pm 0.13$ & $53.03 \pm 0.05$ \\
\hline FRAP assay & $62.3 \pm 0.55$ & $48.6 \pm 0.001$ & $63.6 \pm 0.43$ & $49.8 \pm 0.32$ & $41.3 \pm 0.5$ & $18.2 \pm 0.35$ & $38.3 \pm 0.7$ & $30.2 \pm 0.03$ & $64.2 \pm 0.25$ & $52.22 \pm 0.44$ \\
\hline \multicolumn{11}{|l|}{$\begin{array}{c}\text { NO scavenging } \\
\text { activity }\end{array}$} \\
\hline $30 \mathrm{~min}$ & $32.3 \pm 0.64$ & $10.2 \pm 0.02$ & $21.3 \pm 0.34$ & $10.8 \pm 0.01$ & $8.3 \pm 0.4$ & $2.1 \pm 0.02$ & $9.3 \pm 0.43$ & $6.2 \pm 0.05$ & $33.4 \pm 0.54$ & $15.01 \pm 0.21$ \\
\hline $60 \mathrm{~min}$ & $40.2 \pm 0.23$ & $20.3 \pm 0.21$ & $32.6 \pm 0.1$ & $17.3 \pm 0.01$ & $11.3 \pm 0.2$ & $4.2 \pm 0.31$ & $15.2 \pm 0.2$ & $12.3 \pm 0.8$ & $42.3 \pm 0.07$ & $22.8 \pm 0.31$ \\
\hline $90 \mathrm{~min}$ & $50.2 \pm 0.4$ & $31.4 \pm 0.034$ & $45.3 \pm 0.65$ & $21.2 \pm 0.01$ & $20.1 \pm 0.32$ & $9.3 \pm 0.011$ & $21.2 \pm 0.54$ & $20.4 \pm 0.1$ & $50.4 \pm 0.19$ & $32.06 \pm 0.36$ \\
\hline $120 \mathrm{~min}$ & $53.7 \pm 0.3$ & $40.3 \pm 0.51$ & $50.3 \pm 0.43$ & $28.3 \pm 0.06$ & $25.3 \pm 0.3$ & $13.4 \pm 0.01$ & $30.3 \pm 0.65$ & $25.3 \pm 0.02$ & $58.3 \pm 0.26$ & $42.98 \pm 0.34$ \\
\hline $180 \mathrm{~min}$ & $60.4 \pm 0.11$ & $45.8 \pm 0.021$ & $59.2 \pm 0.21$ & $32.6 \pm 0.055$ & $30.3 \pm 0.43$ & $15.4 \pm 0.003$ & $32.4 \pm 0.5$ & $27.3 \pm 0.1$ & $64.4 \pm 0.17$ & $51.32 \pm 0.23$ \\
\hline TPC (mg/ml) & $10.11 \pm 0.4$ & $12.03 \pm 0.33$ & $13.83 \pm 0.45$ & $12.4 \pm 0.41$ & $8.7 \pm 0.5$ & $2.4 \pm 0.02$ & $7.28 \pm 0.05$ & $6.1 \pm 0.42$ & $16.74 \pm 0.02$ & $13.05 \pm 0.08$ \\
\hline
\end{tabular}

DPPH-1.1-diphenyl -2-picryl hydrazyl; FRAP- Ferric reducing antioxidant power; NO-nitric oxide; TPC-total phenolic content 


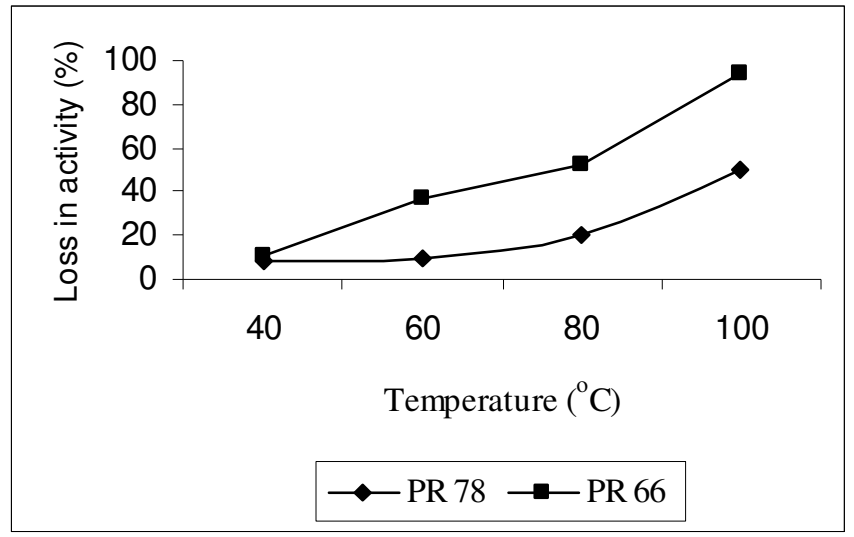

Figure 4. Thermostability of antioxidant potential and total phenolic content of two Aspergillus spp.

Table 4. Effect of extraction with different solvents on antioxidant potential of two Aspergillus spp.

\begin{tabular}{|c|c|c|c|c|c|c|}
\hline \multirow[b]{2}{*}{$\%$ activity } & \multicolumn{2}{|c|}{ Chloroform extract } & \multicolumn{2}{|c|}{ Ethyl acetate extract } & \multicolumn{2}{|c|}{ Butanol extract } \\
\hline & PR78 & PR66 & PR78 & PR66 & PR78 & PR66 \\
\hline DPPH assay & $86.3 \pm 0.09$ & $70.2 \pm 0.1$ & $92.1 \pm 0.1$ & $73.4 \pm 0.3$ & $70.4 \pm 0.2$ & $66.4 \pm 0.03$ \\
\hline Reducing power & $1.53 \pm 0.2$ & $1.42 \pm 0.7$ & $1.92 \pm 0.1$ & $1.73 \pm 0.05$ & $1.04 \pm 0.09$ & $1.01 \pm 0.4$ \\
\hline $\mathrm{Fe}^{2+}$ scavenging activity & $72.4 \pm 0.04$ & $66.2 \pm 0.1$ & $83.4 \pm 0.09$ & $70.2 \pm 0.17$ & $70.2 \pm 0.2$ & $60.4 \pm 0.34$ \\
\hline FRAP assay & $70.4 \pm 0$ & $64.3 \pm 0.1$ & $80.3 \pm 0.1$ & $70.4 \pm 0.05$ & $66.2 \pm 0.02$ & $58.3 \pm 0.45$ \\
\hline \multicolumn{7}{|l|}{ NO scavenging activity } \\
\hline $30 \mathrm{~min}$ & $65.4 \pm 0.06$ & $52.4 \pm 0.2$ & $66.3 \pm 0.09$ & $53.7 \pm 0.2$ & $50.8 \pm 0.3$ & $30.8 \pm 0.23$ \\
\hline $60 \mathrm{~min}$ & $68.9 \pm 0.03$ & $58.3 \pm 0.1$ & $69.0 \pm 0.06$ & $60.4 \pm 0.1$ & $54.3 \pm 0.06$ & $36.4 \pm 0.34$ \\
\hline $90 \mathrm{~min}$ & $70.4 \pm 0.4$ & $60.2 \pm 0.2$ & $72.6 \pm 0.01$ & $62.3 \pm 0.01$ & $58.9 \pm 0.08$ & $42.4 \pm 0.65$ \\
\hline $120 \mathrm{~min}$ & $74.3 \pm 0.02$ & $66.4 \pm 0.3$ & $78.3 \pm 0.3$ & $70.4 \pm 0.1$ & $60.2 \pm 0.01$ & $47.3 \pm 0.05$ \\
\hline $180 \mathrm{~min}$ & $76.3 \pm 0.1$ & $70.1 \pm 0.1$ & $81.4 \pm 0.2$ & $72.4 \pm 0.8$ & $64.3 \pm 0.1$ & $50.4 \pm 0.51$ \\
\hline $\mathrm{TPC}(\mathrm{mg} / \mathrm{ml})$ & $30.76 \pm 0$ & $26.2 \pm 0.1$ & $38.22 \pm 0.3$ & $30.43 \pm 0.4$ & $21.84 \pm 0.1$ & $16.4 \pm 0.009$ \\
\hline
\end{tabular}

DPPH-1.1-diphenyl -2-picryl hydrazyl; FRAP- Ferric reducing antioxidant power;

NO-nitric oxide; TPC-total phenolic content

Table 5. Comparison between different antioxidant assays as represented by correlation coefficient (r).

\begin{tabular}{|c|c|c|c|c|c|}
\hline & DPPH assay & $\begin{array}{l}\text { Reducing } \\
\text { power }\end{array}$ & TPC & $\begin{array}{l}\mathrm{Fe}^{2+} \\
\text { scavenging activity }\end{array}$ & $\begin{array}{l}\text { FRAP } \\
\text { assay }\end{array}$ \\
\hline Reducing power & 0.744 & & & & \\
\hline TPC & 0.614 & 0.903 & & & \\
\hline $\mathrm{Fe}^{2+}$ scavenging activity & 0.784 & 0.951 & 0.772 & & \\
\hline FRAP assay & 0.742 & 0.972 & 0.945 & 0.933 & \\
\hline NO scavenging activity & 0.71 & 0.981 & 0.959 & 0.919 & 0.996 \\
\hline
\end{tabular}


In consonance with earlier studies (26), sucrose proved to be the most promising carbon source to produce bioactive compounds. This explains that a fungal sp may have the ability to utilize a particular carbon source for vegetative growth but may not be able to use it for production of specialized structural molecules. Similarly, sodium nitrate was best nitrogen source among all organic as well as inorganic nitrogen sources, thus indicating that availability of easily utilizable carbon and nitrogen sources promote primary metabolism and feeding with more slowly metabolizable compounds may lead to the formation of secondary products. Nitrogen sources other than sodium nitrate showed lesser activity. This also proves that Czapek dox's medium is most effective for metabolite production responsible for antioxidant activity. In fact culture media, have a major impact on the growth of microbes and the production of microbial products. As far as parameters are concerned there is usually a dilemma between achieving maximal growth rates and maximal bioactivity because conditions that allow fast cell growth could be unfavorable to metabolite production. This shows that the growth medium can also have a significant effect on secondary metabolites and enhancement of secondary metabolites can only be achieved through systematic manipulation of parameters.

Thermostability studies on filtrate demonstrated that metabolites responsible for antioxidant activity are quite stable at $40^{\circ} \mathrm{C}$. Of the different organic solvents extraction tried, ethyl acetate extracts showed best activity correlating with high phenolic content followed by chloroform and butanol extract. Our observations with ethyl acetate extracts are in consonance with the earlier studies $(26,27)$. Further, the results of ethyl acetate extracts were quite comparable with activity of ascorbic acid (96.7 \%), BHA (95.1\%) and alpha tocopherol (94.7\%). The bioactive components may differ in their solubility depending on the extractive solvents used.

Previous studies reveal phenolic compounds to be the major antioxidants of medicinal plants, mushrooms, essential oils, spices, fruits, and vegetables (16). The interest in the phenolic compounds has increased tremendously due to their prominent free radical scavenging activity, attributed to their redox properties, which allow them to act as reducing agents or hydrogen atom donor (2). The importance of phenolic contents has been endorsed from the present observation of their high content in soil fungi and their antioxidant activity is quite comparable with antioxidant potential of many mushrooms as well as medicinal plants.

The comprehensive evaluation of antioxidant activity of natural products using different tests has been shown to be important in assessing antioxidant activity of endogenous compounds. Though it is imperative for researchers to have a convenient, fast, and universal method for overall quantification of antioxidant efficiency of the natural products, however, such a test has yet to be developed. To measure the total antioxidant potential using a single assay procedure seems to be rather unrealistic, yet there are numerous published methods claiming to measure total antioxidant activity in vitro (24).

Thus, to validate the reliability of different methods, the correlation between the different assay procedures is necessary. Previous studies have shown the linear correlation between total phenolic content and antioxidant activity. In this study too, total phenolic content of fungi correlated well with the antioxidant activity. Plotting total phenolic content versus DPPH scavenging activity gave a positive correlation $(\mathrm{r}=0.614)$ between these two parameters indicating the bioactive potential of phenolic compounds which helps to neutralize the free radical character of purple color DPPH either by transfer of electron or hydrogen atom to yellow colored diamagnetic molecule (4).

Similarly a positive correlation was found $(\mathrm{r}=0.903)$ between phenolic content and reducing power where the phenolic compounds in the extracts act as reductones that inhibit lipid peroxidation by donating a hydrogen atom thereby terminating the free radical chain reaction. Moreover, this reducing potential may be due to the di-monohydroxy substitution in the aromatic ring that possesses potent hydrogen donating ability (3).

Nitric oxide exhibits a numerous range of beneficial functions in organisms, including regulation of vascular tone, 
neurotransmission, killing microorganisms, and tumor cells and other homeostatic mechanisms. High levels of nitric oxide have been described in a variety of patho-physiological processes including various forms of circulatory shock, inflammation, and carcinogenesis (1). As evident from studies phenolic compounds present in the extracts are able to scavenge nitric oxide ion as the correlation between them is positive $(r=0.959)$.

Results of FRAP assay are also positively correlated $(\mathrm{r}=0.945)$ with phenolic content showing the latter to have the reducing potential to react with ferric tripyridyl triazine $\left(\mathrm{Fe}^{3+}\right.$-TPTZ) complex and producing blue colored ferrous tripyridyl triazine $\left(\mathrm{Fe}^{2+}-\mathrm{TPTZ}\right)$. Generally the reducing properties are associated by breaking the free radical chain through donating a hydrogen atom (20).

Phenolic compound present in the extracts also showed the chelating activity of metals, as the transition metals such as ferrous ion can stimulate lipid peroxidation by generating hydroxyl radicals through Fenton reaction and accumulate lipid peroxidation by decomposing lipid hydroxyl peroxide into peroxyl and alkoxyl radicals therefore drive the chain reaction of lipid peroxidation. The chelating activity for ferrous ion was assayed by the inhibition of formation of red colored ferrozine and ferrous complex as observed in present studies using both the fungal extracts (29). The correlation between the phenolic content and chelating activity of metals was also positive $(r=0.772)$

The over all positive correlation can be seen between the phenolic content and antioxidant capacity (Table 5). As the total phenolics increases, the antioxidant capacity of the extract from both the Aspergillus spp also increases, indicating that phenolic compound in the extracts are able to scavenge DPPH, ferrous and nitric oxide ion and have reducing potential in addition to their ability to chelate metals such as iron. This indicates that the phenolic content of Aspergillus sp extracts can act as potent antioxidants. These fungi may provide easier set up for production and purification of natural antioxidants as compared to higher plants. The present study demonstrated potential of soil fungi to have antioxidant activity similar to plants and mushrooms thus further highlighting their significance as new sources of natural antioxidants.

\section{REFERENCES}

1. Abas, F.; Lajs, N.H.; Israf, D.A.; Khoziroh, S.; Kalsom, Y.U. (2006). Antioxidant and nitric oxide inhibition activities of selected Malay traditional vegetables. Food Chem., 95, 566-573.

2. Amarowicz, R.; Pegg, R.B.; Moghaddam, P.R.; Barl, B.; Weil, J.A. (2004). Free radical scavenging capacity and antioxidant activity of selected plants species from the Canadian prairies. Food Chem., 84, 551562.

3. Benzie, I.F.; Strain, J.J. (1999). Ferric reducing antioxidant power assay: direct measure of total antioxidant activity of biological fluids and modified version for simultaneous measurement of total antioxidant power and ascorbic acid concentration. Method Enzymol., 299, 15-27.

4. Bounatirou, S.; Smiti, S.; Miguel, M.G.; Falerio, L.; Rejeb, M.N.; Neffati, M.; Casto, M.M.; Figueiredo, A.C.; Barroso, J.G.; Pedro, L.G. (2007). Chemical composition, antioxidant and antibacterial activities of the essential oils isolated from Tunisian Thymus capitatus Hoff. et link. Food Chem., 105, 146-155.

5. Chang, L.W.; Yen, W.J.; Huang, S.C.; Duh, P. D. (2002). Antioxidant activity of sesame coat. Food Chem., 78, 347-354.

6. Fox, E.M.; Howlett, J.B. (2008). Secondary metabolism: regulation and role in fungal biology .Curr. Opin. Microbiol., 11, 1-7.

7. Harper, K.; Arif, A.; Ford, E.J.; Strobel, G.A.; Porco Jr, J.A.; Tomar, D.P.; Oneill, K.L.; Heider, E.M.; Grant, D.M., (2003). Pestacin: a 1,3dihydro isobenzofuran from Pestalotiopsis micropora possessing antioxidant and antimycotic activities. Tetrahedron, 59, 2471-2476.

8. Huang, D.J.; Chen, H.J.; Hou, W. C.; Lin, C.D.; Lin, Y.H. (2006). Sweet potato (Ipomoea batatas [L.] Lam 'Tainong 57') storage root mucilage with antioxidant activities in vitro. Food Chem., 98, 774-781.

9. Huang, W.Y.; Cai, Y.Z.; Hyde, K.D.; Corke, H.; Sun, M. (2007). Endophytic fungi from Nerium oleander L (Apocynaceae): main constituents and antioxidant activity. World J. Microbiol. Biotechnol., 23, 1253-1263.

10. Kang, K.S.; Yokozawa, T.; Kim, H.Y.; Park, J.H. (2006). Study on the nitric oxide scavenging effects of Gingseng and its compound. J. Agric. Food. Chem., 54, 2558-2562.

11. Lucas, E.M.F.; De Castro, M.C.M.; Takahashi, J.A. (2007). Antimicrobial properties of sclerotiorin, isochromophilone vi and pencolide, metabolites from a Brazilian Cerrado isolate of Penicillium sclerotiorum van beyma. Brazilian J. Microbiol., 38,785-789.

12. Malpure, P.P.; Shah, A.S.; Juvekar, A.R. (2006). Antioxidant and antiinflammatory activity of extract obtained from Aspergillus candidus MTCC 2202 broth filtrate. Indian J. Exp. Biol., 44, 468-473.

13. Mathew, S.; Abraham, T.E. (2006). Studies on the antioxidant activities of Cinnamon (Cinnamomum verum) bark extracts, through various in 
vitro models. Food Chem., 94, 520-528.

14. Miao, L.; Kwong, T.F.N.; Qian, P.Y. (2006). Effect of culture conditions on mycelial growth, antibacterial activity, and metabolite profiles of the marine-derived fungus Arthrinium c.f. saccharicola. Appl. Microbiol. Biotechnol., 72, 1063-1073.

15. Miller, M. J.; Diplock, A. T.; Rice-Evans, C.A. (1995). Evaluation of the total antioxidant activity as a marker of the deterioration of apple juice on storage. J. Agric. Food Chem., 43, 1794-1801.

16. Mokbel, M. S.; Hashinaga, F. (2006). Evaluation of the antioxidant activity of extracts from Buntan (Citrus grandis Osbeck) fruit tissues. Food Chem., 94, 529-534.

17. Moon, B.S.; Ryoo, I.J.; Yun, B. S.; Bae, K.S.; Lee, K.D.; Yoo, I.D.; Kim, J.P. (2006). Glyscavins A,B and C, new phenolic glycoside antioxidants produced by a fungus Mycelia sterilia F020054. J. Antibiot., 59, 735-739.

18. Motta, A.S.; Cladera-Olivera, F.; Brandelli, B. (2004). Screening for antimicrobial activity among bacteria isolated from the Amazon basin. Braz. J. Microbiol., 35, 307-310.

19. Nogurira, M.A.; Diaz, G.; Andriali, W.; Faiconi, A.F.; Stangarlin, S.R. (2006). Secondary metabolism from Diplodia maydis and Sclerotium rolfsi with antibiotic activity. Braz. J. Microbiol., 37, 14-16.

20. Othman, A.; Ismail, A.; Ghani, N.A.; Adenan, I. (2007). Antioxidant capacity and phenolic content of cocoa beans.Food Chem., 100, 15231530.

21. Rios, M.F.; Pajan, C.M.G.; Galan, R.H.; Sanchez, A.J.M.; Callado, I.G.
(2006). Synthesis and free radical scavenging activity of a novel metabolite from the fungus Colletotrichum gloeosporioides. Bioorg. Med. Chem. Lett., 16, 5836-5839.

22. Singleton, V.L.; Ortofehr, R.; Lamuela-Raventos, R.M. (1999). Analysis of total phenols and other oxidation substrate and antioxidants by means of Folin-Ciocalteau reagent. Methods Enzymol., 299,152-178.

23. Song, T.Y.; Yen, G.C. (2002). Antioxidant properties of Antrodia camphorata in submerged culture. J. Agri. Food Chem., 50, 3322-3327.

24. Sultana, B.; Anwar, F.; Przybylski, R. (2007). Antioxidant activity of phenolic components present in barks of Azadirachta indica, Terminalia arjuna, Acacia nilotica and Eugenia jambalana Lam.trees. Food Chem., 104, 1106-1114.

25. Yen, G.C.; Lee, C.A. (1996). Antioxidant activity of extracts from molds. J.Food Prot., 59, 1327-1330.

26. Yen, G.C.; Chang, Y.C. (1999). Medium optimization for the production of antioxidants from Aspergillus candidus. J. Food Prot., 62, 657-661.

27. Yen, G.C.; Chang, Y.C.; Sheu, F.; Chiang, H.C. (2001). Isolation and characterization of antioxidants compounds from Aspergillus candidus broth filtrate. J. Agri. Food Chem., 49, 1426-1431.

28. Yhu, Z.; Pan, J.; Qiu, J.; Guan, X. (2008). Optimization of nutritional requirements for mycelial growth and sporulation of entomogenous fungus Aschersonia aleyrodis webber. Braz. J. Microbiol., 39,770-775.

29. Zhao, G.R.; Xiang, Z.J.; Ye, T.X.; Yaun, J.Y.; Guo, X.Z. (2006). Antioxidant activities of Salvia miltiorrhiza and Panax notoginseng. Food Chem., 99, 767-774. 